\section{The influence of posterior capsule opacification on scanning laser polarimetry}

M Vetrugno', F Masselli', G Greco', D Sisto1, A Maino $^{2}$, S Ficarelli ${ }^{1}$ and $G$ Sborgia ${ }^{1}$
${ }^{1}$ Department of Ophthalmology and Otolaryngology, University of Bari, Bari, Italy

${ }^{2}$ Royal Eye Hospital, Manchester, UK

Correspondence: M Vetrugno, Dipartimento di Oftalmologia e Otorinolaringoiatria, Azienda Ospedaliera 'Policlinico', Piazza Giulio Cesare 11, 70124 Bari, Italy Tel: + 30080 5592525; Fax: + 390805478918 E-mail: glaucoma@ oftalmo.uniba.it

Received: 22 June 2005 Accepted in revised form: 28 January 2006 Published online: 7 April 2006

Competing interests: The authors have no financial or other interests in any of the products mentioned

\begin{abstract}
Purpose To evaluate the influence of posterior capsule opacification (PCO) on GDx parameters in a population of pseudophakic, non-glaucomatous patients who underwent Nd:YAG laser capsulotomy (YLC).

Methods The posterior capsules were photographed with a Topcon digital camera and each image was then entered into the EPCO 2000 software and evaluated independently by three examiners. The EPCO 2000 software was used to calculate the fibrosis index (FI) and the pearl index (PI) for the central 1.5, 2.5, and $3.5 \mathrm{~mm}$ of the posterior capsule. Scanning laser polarimetry was performed with GDx before and after YLC. We compared the GDx readings obtained before and after the YLC using paired Student's $t$-test. The parameters that varied significantly after YLC were subsequently used for regression analysis. Stepwise multiple linear regression was used to analyse the impact of the change in the amount of FI and PI on change in GDx parameters after YLC.

Results In total, 158 patients were enrolled ( 74 men, 84 women). The mean age was $69.46 \pm 8.83$ years (range $46-83$ years). The interobserver agreement among the three experts was found to be good (repeatability coefficient $R=1.51,1.49,1.49$ for observer $\mathrm{A}$ vs $\mathbf{B}, \mathbf{A}$ vs $\mathbf{C}$, and $\mathbf{B}$ vs $\mathbf{C}$ respectively). One-sample Student's $t$-test show no difference between all GDx parameters before and after YLC except for Symmetry, Superior/Nasal ratio, Inferior Ratio, and Temporal-Superior-Nasal-InferiorTemporal (TSNIT). Stepwise multiple regression showed that the two variables of greatest significance for changes in Symmetry were the FI in the central 1.5 and the PI in the central $3.5 \mathrm{~mm}(P=0.02)$. Superior/nasal ratio was shown to be most strongly correlated to the FI in the central $1.5 \mathrm{~mm}$ and PI in the
\end{abstract}

central $3.5 \mathrm{~mm}(P<0.001)$, whereas the variable of greatest significance to Inferior Ratio was PI in the central $3.5 \mathrm{~mm}(P=0.03)$. Finally, TSNIT was most strongly correlated to FI in the central $1.5 \mathrm{~mm}$ and FI in the central $2.5 \mathrm{~mm}$ $(P<0.001)$.

Conclusion Presence of capsular fibrosis seems to be more clinically relevant in the central zone, whereas pearls tend to be clinically significant in the central $3.5 \mathrm{~mm}$ area. Hence, it might be worthwhile assessing the amount of PCO in pseudophakic patients when performing scanning laser polarimetry. Investigators should ensure that the type of PCO and the size of the area analysed are documented in the notes in order to interpret GDx findings appropriately.

Eye (2007) 21, 760-763; doi:10.1038/sj.eye.6702323; published online 7 April 2006

Keywords: scanning laser polarimetry; posterior capsule opacification; laser capsulotomy

\section{Introduction}

Despite advances in cataract surgery and intraocular lens design, posterior capsular opacification (PCO) is the commonest complication of cataract surgery. ${ }^{1}$ Opacification of the posterior capsule can lead to clinically significant reduction in visual acuity, impaired contrast sensitivity, glare disability, and monocular diplopia. ${ }^{2,3}$ Moreover, PCO can have a significant impact on the ability to visualize the posterior segment and, as many diagnostic devices used in ophthalmology rely on a clear visual axis, severe PCO might interfere with data acquisition and lead to an incorrect or delayed diagnosis. In particular, this could be especially important for patient undergoing scanning laser polarimetry with GDx, an increasingly popular method used for the diagnosis of early glaucoma. 
To date, little is known about the influence of PCO on GDx parameters. Our study compares how the GDx parameters change after YAG laser capsulotomy (YLC), taking into account three different areas of the posterior capsule (namely, central 1.5, 2.5, and $3.5 \mathrm{~mm}$ ).

\section{Methods}

All patients attending the Department of Ophthalmology to undergo Nd:YAG laser capsulotomy for the first time were eligible for this study. Exclusion criteria were age below 18 years, presence of optic nerve pathologies (including glaucoma), refractive error higher than \pm 4 spherical dioptres and three cylindrical dioptres, retinal pathologies, previous ophthalmic surgery other than cataract surgery in the first eye, visual acuity less than 1.0 LogMAR, GDx score of 8 or less, PCO grade 4 (EPOC system), participation in other trials, and refusal to participate. The study was approved by the regional Ethical Committee. A total of 158 patients met the abovementioned criteria and were divided into two groups: 128 patients entered the main study, whereas 30 were selected for an ancillary agreement study (see flowchart in Figure 1). None of the experts operating the analysis system was informed about the intent of the additional 30 patients.

Baseline best-corrected visual acuity (BCVA) was assessed using LogMAR charts. Scanning laser polarimetry was performed on each patient using GDx Access (Laser Diagnostic Technologies, San Diego, CA, USA). All GDx acquisitions were taken by the same investigator (SF). The following RNFL parameters were measured: NFI, Symmetry, Superior Ratio, Inferior Ratio, Superior/Nasal, Max Modulation, Superior Maximum, Inferior Maximum, Ellipse Modulation, Normalized Superior Area, Normalized Inferior Area, Ellipse standard deviation, Ellipse Average, Superior Average, Inferior Average,and Temporal-Superior-Nasal-InferiorTemporal (TSNIT) standard deviation.

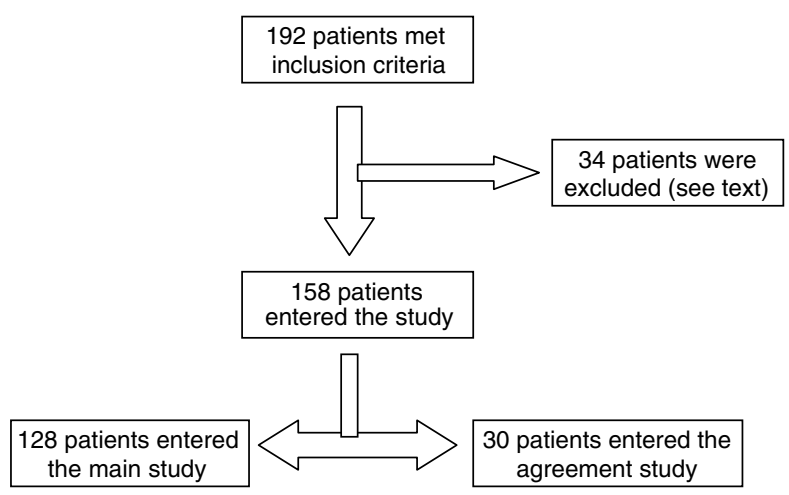

Figure 1 Patients allocation (flow chart).
Patients' pupillary diameter was measured before instilling $0.5 \%$ tropicamide drops. The posterior capsules were photographed with a Topcon digital camera using the Imagenet 2000 software system (Topcon Corporation, Tokyo, Japan). Each image was then entered into the EPCO 2000 image analysis software (EPCO 2000, (c) Ch. Nimsgern, 2000; www.epco2000.de) and evaluated independently by three examiners (MV, FM, DS).

The EPCO 2000 software was used to demarcate the areas of pearls separately from those of fibrosis and calculate the fibrosis index (FI) and the pearl index (PI) for the central 1.5, 2.5, and $3.5 \mathrm{~mm}$ of the posterior capsule. A detailed description of the EPOC software and the calculations is available elsewhere. ${ }^{4,5}$

Following this preliminary assessment, the patients underwent Nd:YAG capsulotomy by a surgeon (MV) using a standardized protocol. The eyes were assigned to the treatment/control groups randomly, according to a computer-generated list. In the treatment group, the energy level was set at $1 \mathrm{~mJ}$ and subsequent incremental increases of $0.5 \mathrm{~mJ}$ were made until the posterior capsule was penetrated. The total amount of energy ranged from 2.4 to $5.3 \mathrm{~mJ}$ fractioned in 3-5 shots in a cruciate pattern, hence creating an opening of about $3 \mathrm{~mm}$. Patients returned 1 week later and had repeat testing of BCVA and scanning laser polarimetry as described above. Control eyes underwent all the above measurements 1 week later.

Interobserver agreement was assessed by the repeatability coefficient among three experts. The repeatability coefficient describes the limit of agreement, that is, the maximum difference in the results that can be expected in $95 \%$ of cases.

We compared the GDx readings obtained before and after the YLC using paired Student's $t$-test. The parameters that varied significantly after YLC were subsequently used for regression analysis. Stepwise multiple linear regression was used to analyse the impact of the change in the amount of fibrosis (FI) and pearls (PI) on change in GDx parameters after YLC.

\section{Results}

In total, 158 patients were enrolled ( 74 men, 84 women). The mean age was $69.46 \pm 8.83$ years (range $46-83$ years). The average time elapsed from the cataract surgery and the YLC was $31.42 \pm 27.22$ months (range 1-120 months). The average (undilated) pupillary diameter was $2.7 \pm 0.3 \mathrm{~mm}$. Mean visual acuity was $0.3 \pm 0.6$ Logmar (before YLC) and 0.05 \pm 0.2 Logmar (after YLC) $(P<0.001)$. Based upon the EPCO analysis system, 46 had PCO grade 3, 34 had grade 2, and 48 had grade 1 . The interobserver agreement among the three experts was found to be good (repeatability coefficient $R=1.51,1.49$, 
and 1.49 for observer A vs B, A vs C, and B vs C, respectively). One-sample Student's $t$-tests show no difference between all GDx parameters before and after YLC except for Symmetry, Superior/Nasal ratio, Inferior Ratio, and TSNIT as shown in Table 1. Data from the control group are displayed in Table 2. Similarly, onesample Student's $t$-test show no difference between treatment and control groups when FI and PI scores $(1.5,2.5$ and $3.5 \mathrm{~mm})$ are compared, as shown in Tables 3 and 4 .

The first variable to be studied was change in the symmetry parameter. Stepwise multiple regression showed that the two variables of greatest significance were the FI in the central 1.5 and the PI in the central

Table 1 GDx parameter changes in the treatment group

\begin{tabular}{lccl}
\hline & $\begin{array}{c}\text { Pre-NYLC } \\
(\text { mean } \pm S D)\end{array}$ & $\begin{array}{c}\text { Post-NYLC } \\
(\text { mean } \pm S D)\end{array}$ & P-value \\
\hline The Number & $23.27 \pm 18.01$ & $24.19 \pm 19.81$ & 0.72 \\
Symmetry & $\mathbf{0 . 9 5} \pm \mathbf{0 . 1 7}$ & $\mathbf{1 . 2 5} \pm \mathbf{0 . 2 4}$ & $\mathbf{0 . 0 0 0 1}$ \\
Superior Ratio & $2.10 \pm 1.11$ & $1.95 \pm 0.93$ & 0.29 \\
Inferior Ratio & $\mathbf{2 . 2 3} \pm \mathbf{1 . 1 0}$ & $\mathbf{1 . 9 3} \pm \mathbf{0 . 8 8}$ & $\mathbf{0 . 0 3}$ \\
Superior/Nasal & $\mathbf{1 . 7 4} \pm \mathbf{0 . 6 9}$ & $\mathbf{1 . 9 6} \pm \mathbf{0 . 7 1}$ & $\mathbf{0 . 0 2}$ \\
Max Modulation & $1.45 \pm 1.08$ & $1.26 \pm 0.95$ & 0.17 \\
Superior Maximum & $97.83 \pm 40.98$ & $94.11 \pm 34.85$ & 0.48 \\
Inferior Maximum & $100.49 \pm 35.45$ & $93.24 \pm 33.55$ & 0.17 \\
Ellipse Modulation & $2.74 \pm 1.58$ & $2.36 \pm 1.26$ & 0.05 \\
Normalized Sup. Area & $0.15 \pm 0.09$ & $0.16 \pm 0.08$ & 0.39 \\
Normalized Inf. Area & $0.16 \pm 0.08$ & $0.16 \pm 0.08$ & 0.99 \\
Ellipse Std. Dev. & $22.84 \pm 13.60$ & $20.17 \pm 8.17$ & 0.08 \\
Ellipse Average & $68.35 \pm 24.48$ & $69.68 \pm 30.47$ & 0.72 \\
Superior Average & $73.18 \pm 27.36$ & $77.27 \pm 28.25$ & 0.29 \\
Inferior Average & $73.18 \pm 24.77$ & $73.32 \pm 27.80$ & 0.96 \\
TSNIT Std. Dev. & $23.22 \pm 13.11$ & $20.18 \pm 8.17$ & $\mathbf{0 . 0 4}$ \\
\hline
\end{tabular}

Bold indicate statistically significant values.

Table 2 GDx parameter changes in the control group

\begin{tabular}{lccc}
\hline & $\begin{array}{c}\text { Pre-YLC } \\
(\text { mean } \pm S D)\end{array}$ & $\begin{array}{c}\text { Post-YLC } \\
(\text { mean } \pm S D)\end{array}$ & P-value \\
\hline NFI & $28.71 \pm 19.05$ & $28.29 \pm 19.43$ & 0.87 \\
Symmetry & $0.99 \pm 0.14$ & $1.01 \pm 0.16$ & 0.33 \\
Superior Ratio & $2.16 \pm 0.83$ & $2.28 \pm 0.90$ & 0.31 \\
Inferior Ratio & $2.17 \pm 0.80$ & $2.24 \pm 0.84$ & 0.53 \\
Superior/Nasal & $2.16 \pm 0.78$ & $2.15 \pm 0.70$ & 0.92 \\
Max Modulation & $1.59 \pm 0.89$ & $1.68 \pm 0.77$ & 0.43 \\
Superior Maximum & $81.24 \pm 21.92$ & $83.24 \pm 22.38$ & 0.51 \\
Inferior Maximum & $82.95 \pm 25.64$ & $83.89 \pm 26.50$ & 0.79 \\
Ellipse Modulation & $2.44 \pm 1.05$ & $2.64 \pm 1.14$ & 0.18 \\
Normalized Sup. Area & $0.13 \pm 0.05$ & $0.14 \pm 0.05$ & 0.15 \\
Normalized Inf. Area & $0.14 \pm 0.06$ & $0.14 \pm 0.06$ & 0.99 \\
Ellipse Std. Dev. & $18.75 \pm 6.02$ & $19.62 \pm 6.56$ & 0.32 \\
Ellipse Average & $60.14 \pm 19.56$ & $60.99 \pm 20.21$ & 0.75 \\
Superior Average & $66.58 \pm 17.54$ & $67.61 \pm 18.40$ & 0.67 \\
Inferior Average & $67.47 \pm 23.65$ & $68.25 \pm 24.15$ & 0.81 \\
TSNIT Std. Dev. & $18.75 \pm 6.02$ & $19.62 \pm 6.56$ & 0.32 \\
\hline
\end{tabular}

$3.5 \mathrm{~mm}$. All other variables were excluded by the backward stepwise analysis, as they were not found to add further to the final result. The overall regression correlation was $0.74(P=0.02)$.

Similarly, Superior/nasal ratio was shown to be most strongly correlated to the FI in the central $1.5 \mathrm{~mm}$ and PI in the central $3.5 \mathrm{~mm}$ (overall regression correlation $=0.667, P<0.001$ ), whereas the variable of greatest significance to Inferior Ratio was PI in the central $3.5 \mathrm{~mm}$ (overall regression correlation $=0.71, P=0.03$ ). Finally, TSNIT was most strongly correlated to FI in the central $1.5 \mathrm{~mm}$ and FI in the central $2.5 \mathrm{~mm}$ (overall regression correlation $=0.77, P<0.001$ )

\section{Discussion}

Posterior capsule opacification morphology affects vision and contrast sensitivity. ${ }^{6}$ Several studies have analysed the impact of posterior capsule fibrosis and the presence of Elschnig's pearls on visual acuity. The general consensus is that pearls could affect visual acuity by scattering light, whereas fibrosis tends to be significantly associated with decreased visual acuity in central zones. Aslam et $a l^{4}$ identified the presence of fibrosis in the central $1.5 \mathrm{~mm}$ area and the presence of pearls in the central $3.5 \mathrm{~mm}$ area as the most clinically relevant factors determining visual acuity and contrast sensitivity. Our study shows that, out of the four parameters that varied

Table 3 Changes in pearl and fibrosis indexes in the treatment group

\begin{tabular}{lccc}
\hline Parameters & Pre-NYLC & Post-NYLC & P-value \\
\hline FI $1.5 \mathrm{~mm}$ & $1.8 \pm 0.4$ & $0.3 \pm 0.6$ & $<0.001$ \\
FI $2.5 \mathrm{~mm}$ & $1.9 \pm 0.6$ & $0.4 \pm 0.4$ & $<0.001$ \\
FI $3.5 \mathrm{~mm}$ & $2.4 \pm 0.6$ & $0.7 \pm 0.4$ & $<0.001$ \\
PI $1.5 \mathrm{~mm}$ & $0.8 \pm 0.2$ & $0.4 \pm 0.2$ & $<0.001$ \\
PI $2.5 \mathrm{~mm}$ & $0.7 \pm 0.3$ & $0.4 \pm 0.2$ & $<0.001$ \\
PI $3.5 \mathrm{~mm}$ & $0.5 \pm 0.2$ & $0.2 \pm 0.3$ & $<0.001$ \\
\hline
\end{tabular}

FI, fibrosis index; PI, pearl index.

Table 4 Changes in pearl and fibrosis indexes in the control group

\begin{tabular}{lccc}
\hline Parameters & Pre-NYLC & Post-NYLC & P-value \\
\hline FI $1.5 \mathrm{~mm}$ & $1.8 \pm 0.4$ & $0.3 \pm 0.6$ & $<0.001$ \\
FI $2.5 \mathrm{~mm}$ & $1.9 \pm 0.6$ & $0.4 \pm 0.4$ & $<0.001$ \\
FI $3.5 \mathrm{~mm}$ & $2.4 \pm 0.6$ & $0.7 \pm 0.4$ & $<0.001$ \\
PI $1.5 \mathrm{~mm}$ & $0.8 \pm 0.2$ & $0.4 \pm 0.2$ & $<0.001$ \\
PI $2.5 \mathrm{~mm}$ & $0.7 \pm 0.3$ & $0.4 \pm 0.2$ & $<0.001$ \\
PI $3.5 \mathrm{~mm}$ & $0.5 \pm 0.2$ & $0.2 \pm 0.3$ & $<0.001$ \\
\hline
\end{tabular}

FI, fibrosis index; PI, pearl index. 
significantly after YLC, three parameters (Symmetry, Superior/Nasal, and TSNIT) were influenced by the presence of fibrosis in the central $1.5 \mathrm{~mm}$ diameter area and three (Symmetry, Superior Nasal, and Inferior Ratio) were significantly influenced by the presence of Elschnig's pearls in a more peripheral position (central $3.5 \mathrm{~mm}$ area). This is in line with previous studies ${ }^{4}$ and we might assume that fibrosis seems to be more clinically relevant in the central zone, whereas pearls tend to affect GDx parameters significantly only in a more peripheral area of $3.5 \mathrm{~mm}$ diameter.

It might be hypothesized that global parameters tend to be more significantly influenced by fibrosis, which is usually distributed across a large area, whereas parameters like Inferior Ratio, which are obtained by comparing different zones of the retina, might be more susceptible to localized changes, such as Elschnig's pearls. On the other hand, our patients might not represent an adequate sample. By enrolling those awaiting YLC, we might have included a higher than average proportion of patient with clinically significant central PCO or eccentric pearls. ${ }^{7,8}$ It might be interesting repeating the study on a group of asymptomatic patients.

A limitation of our study is the subjective method for quantifying posterior capsule opacification. EPOC 2000 relies on the examiner's judgement for the delimitation of the area to be analysed and therefore is subject to errors. Even though the software has been widely accepted as a reliable research tool, especially as other methods are less readily available, we tried to reduce observational bias by enrolling a panel of three experts that processed the images independently. ${ }^{9-11}$ Another confounding factor could be the intrinsic variability of the GDx parameters.

Rhee et al conducted a study on pseudophakic patients, which was aimed to assess the reproducibility of retinal fibre layer measurements using GDx. It was found that GDx measurements can be obtained with good reproducibility, perhaps with the exception of Ellipse Modulation and the Number. ${ }^{12}$ Our study showed how both these parameters did not vary significantly after YLC whereas the four parameters that were significantly altered by YLC in our study (Symmetry, Superior/Nasal, Inferior Ratio, TSNIT) were reproducible, with coefficients of variation of $10 \%$ or less.
Hence, it might be argued that our results are not affected by random variations.

In conclusion, it might be worthwhile assessing the amount of PCO in pseudophakic patients when performing scanning laser polarimetry. Investigators should ensure that the type of PCO and the size of the area analysed are documented in the notes in order to grant the GDx findings clinical relevance.

\section{References}

1 Wormstone IM. Posterior capsule opacification: a cell biological perspective. Exp Eye Res 2002; 74: 337-347.

2 Claesson MKL, Bechman C. Glare and contrast sensitivity before and after Nd:YAG laser capsulotomy. Acta Ophthalmol 1994; 72: 27-32.

3 Sunderraj P, Villada JR, Joyce PW, Watson A. Glare testing in pseudophakes with posterior capsule opacification. Eye 1992; 6(Part 4): 411-413.

4 Aslam TM, Aspinall P, Dhillon B. Posterior capsule morphology determinants of visual function. Graefe's Arch Clin Exp Ophthalmol 2003; 241: 208-212.

5 Tetz MR, Auffarth GU, Sperker M, Blum H, Volcker HE. Photographic image analysis system of posterior capsule opacification. J Cataract Refract Surg 1997; 23(10): 1515-1520.

6 Cheng CY, Yen MY, Chen SJ et al. Visual acuity and constrast sensitivity in different types of posterior capsule opacification. J Cataract Refract Surg 2001; 27(7): 1055-1060.

7 Patton N, Aslam TM, Bennett HG et al. Does a small central Nd:YAG posterior capsulotomy improve peripheral fundal visualisation for the vitreoretinal surgeon? BMC Ophthalmol 2004; 4(1): 8 .

8 Aslam TM, Dhillon B, Werghi N et al. Systems of analysis of posterior capsule opacification. $\mathrm{Br}$ J Ophthalmol 2002; 86: 1181-1186.

9 Barman SA, Hollick EJ, Boyce JF et al. Quantification of posterior capsular opacification in digital images after cataract surgery. Invest Ophthalmol Vis Sci 2000; 41(12): 3882-3892.

10 Findl O, Buehl W, Menapace R et al. Comparison of 4 methods for quantifying posterior capsule opacification. J Cataract Refract Surg 2003; 29: 106-111.

11 Aslam TM, Patton N, Graham J. A freely accessible, evidence based, objective system of analysis of posterior capsular opacification; evidence for its validity and reliability. BMC Ophthalmol 2005; 5(1): 9.

12 Rhee DJ, Greenfield DS, Chen PP, Schiffman J. Reproducibility of retinal nerve fiber layer thickness measurements using scanning laser polarimetry in pseudophakic eyes. Ophthalmic Surg Lasers 2002; 33(2): 117-122. 